\title{
Answers Regarding the Etiology of Severe Community-Acquired Pneumonia: Usefulness of Polymerase Chain Reaction Techniques
}

\author{
Alessandro Vatrella \\ Department of Medicine, Surgery and Dentistry 'Scuola Medica Salernitana', University of Salerno, Baronissi, Italy
}

Community-acquired pneumonia (CAP) represents an important cause of morbidity and mortality worldwide and is a major cause of hospitalization both in children and adults [1]. The etiology of CAP, in particular in the severe forms, is complex and includes many different bacteria and viruses. An accurate and rapid detection of pathogens directly affects decisions regarding the treatment of patients with CAP, which is crucial in achieving the best clinical management, and international guidelines recommend increased efforts to identify the causative agents in severe CAP.

Traditional diagnostic methods which primarily employ cultures often fail to identify the causative organisms, and calls have been made for greater development and use of more sensitive and rapid molecular tests. Polymerase chain reaction (PCR) and real-time PCR are emerging technologies for the detection and quantification of respiratory pathogens and have been demonstrated to improve the diagnosis of viral and bacterial respiratory infections [2]. Most studies using PCR techniques for pathogen detection have involved severe sepsis and bloodstream infections in general pneumonia rather than CAP. The few that have used PCR for viruses in severe $\mathrm{CAP}$ were performed in temperate countries and yielded disparate results [3].

The article by Siow et al. [4], published in this issue of Respiration, assessed in a large prospective study if the use of PCR techniques, for both viral and bacterial pathogens, may improve the detection of pathogens in patients admitted to the intensive care unit with severe CAP. Virus- es and bacteria were detected in 7 of 10 patients with severe CAP. PCR amplification was able to increase the detection of pathogens from 42 to $69 \%$ of patients, of which $37 \%$ had bacterial infection, $14 \%$ had viral infection, and $18 \%$ had bacterial-viral coinfection. These results suggest that a large proportion of patients with severe CAP in a tropical setting who would previously have been considered culture negative are in fact infected by various viruses and/or bacteria.

In conclusion, the use of PCR analysis could improve the ability to detect both viral and bacterial pathogens in severe CAP, providing clinicians with answers regarding etiology. The implementation of PCR tests in clinical practice may provide the opportunity to identify the etiological agent in a clinically relevant time period, may allow more precise antibiotic prescription, and may aid in differentiating true infection from colonization and contamination.

\section{References}

1 Wunderink RG, Waterer GW: Clinical practice. Community-acquired pneumonia. N Engl J Med 2014;370:543-551.

2 Zumla A, Al-Tawfiq JA, Enne VI, et al: Rapid point of care diagnostic tests for viral and bacterial respiratory tract infections: needs, advances, and future prospects. Lancet Infect Dis 2014;14:1123-1135.

- 3 Wu X, Wang Q, Shi Y, et al: Incidence of respiratory viral infections detected by PCR and real-time PCR in adult patients with communityacquired pneumonia: a meta-analysis. Respiration 2015;89:343-352.

4 Siow WT, Koay ES-C, Lee CK, et al: The use of polymerase chain reaction amplification for the detection of viruses and bacteria in severe community-acquired pneumonia. Respiration 2016;92:286-294.

\section{KARGER}

E-Mail karger@karger.com

www.karger.com/res
C 2016 S. Karger AG, Basel

0025-7931/16/0925-0285\$39.50/0
Prof. Alessandro Vatrella, MD

Department of Medicine, Surgery and Dentistry 'Scuola Medica Salernitana' University of Salerno, Baronissi Campus, Via S. Allende IT-84081 Baronissi (Italy)

E-Mail avatrella@unisa.it 